\title{
Slow Gamma Activity of Local Field Potentials (LFP) in the Freely Moving Rat Relates to Movement
}

\author{
Wilfried Dimpfel1, Leonie Schombert ${ }^{2}$ \\ ${ }^{1}$ Justus-Liebig-Unversität-Giessen, Giessen, Germany \\ ${ }^{2}$ NeuroCode AG, Wetzlar, Germany \\ Email: w.dimpfel@neurocode-ag.com
}

Received 21 July 2015; accepted 6 September 2015; published 10 September 2015

Copyright (C) 2015 by authors and Scientific Research Publishing Inc.

This work is licensed under the Creative Commons Attribution International License (CC BY). http://creativecommons.org/licenses/by/4.0/

(c) (i) Open Access

\begin{abstract}
Quantitative assessment of local field potentials by means of Fast Fourier Transformation (FFT) results in the so-called power density spectrum. Within this spectrum particular frequency ranges are defined in order to relate these to behavior. Frequencies above $35 \mathrm{~Hz}$ are generally labeled as gamma oscillations, especially as low gamma (40 - 55 Hz) or high gamma $(70-100 \mathrm{~Hz})$. In order to learn more about this feature, we implanted a set of 4 bipolar concentric steel electrodes in frontal cortex, hippocampus, striatum and midbrain reticular formation of 10 rats. After recovery, field potentials were recorded and wirelessly transmitted to our computer for frequency analysis. At the same time, motion was registered during the whole experimental period of 5.75 hours. Results revealed that low gamma activity only emerged when the animal moved-at least his head. FFT of the data showed-besides other frequencies-a slow gamma activity peaking around $47 \mathrm{~Hz}$ predominantly within the striatum, less in frontal cortex and reticular formation and nearly none in the hippocampus. Spectral analysis was performed for single epochs of 4 seconds and all 15 minutes intervals. Correlation analysis of these intervals was done to motion data. All rats showed a highly significant correlation between gamma activity and movement. We therefore conclude from these experiments that this slow gamma activity of the field potentials is not only related to movement, but possibly part of the general neuronal coding of movement.
\end{abstract}

\section{Keywords}

Local Field Potential (LFP), Gamma Activity, Rat, Movement 


\section{Introduction}

There are quite a number of papers dealing with so-called gamma activity of local field potentials (LFP) in the literature, for example, as suggested already by Igarashi et al. [1]. Basically, slow gamma activity (around $50 \mathrm{~Hz}$ ) is differentiated from fast gamma activity (around $80 \mathrm{~Hz}$ ) [2]. This activity has been recorded within several brain areas in animals and humans. However, there is no common understanding of the relevance of this fast oscillatory activity with respect to behavior. There are contributions, which relate slow gamma activity to movement [3], others describe probable involvement of gamma activity to attention, learning, memory or decision making. Local relevance of these gamma-oscillations has been proven, since putative fast-spiking interneurons showed phase, firing rate, and coherence relationships with this activity [4].

Since we observed this activity mainly within the striatum during an experimental project aiming at the characterization of new developmental compounds, we took a closer look at this fast oscillation with respect to the four brain areas we were recording from for many years during control experiments after saline injection. The present analysis aimed at the possibility that these oscillations were related to movement, since they were most prominent in recordings from striatum.

\section{Material and Methods}

Adult male Fischer 344 rats (about 400 g bodyweight), obtained from Charles River Laboratories, Sulzfeld, Germany, were implanted with 4 bipolar concentric steel electrodes within a stereotactic surgical procedure during anesthesia with Ketamine [5]. All four electrodes were placed $2.9 \mathrm{~mm}$ lateral within the left hemisphere. Dorso-ventral coordinates were 4, 6, 4.2 and $8 \mathrm{~mm}$ and anterior coordinates were 3.7, 9.7, 5.7 and $12.2 \mathrm{~mm}$ for frontal cortex, striatum, hippocampus, and reticular formation, respectively (according to the atlas of [6]). A preconstructed base plate carrying 4 bipolar stainless steel semi-micro electrodes (neurological electrodes "SNF 100” from Rhodes Medical Instruments, Inc., Summerland, CA, USA) and a 5-pin-plug (Figure 1) was fixed to the skull by dental cement interacting with 3 steel screws placed on distance into the bone. The distant recording spot of the electrode was the active electrode, whereas the proximal spots of the four electrodes were connected to each other to give a short circuit reference. The base plate was carrying a plug to receive later on the transmitter (weight: $5.2 \mathrm{~g}$ including battery, 26_12_6 $\mathrm{mm}^{3}$ of size). EEG signals were recorded from frontal cortex, hippocampus, striatum and midbrain reticular formation of freely moving rats from inside a totally copper shielded room (Figure 2). Signals were wirelessly transmitted by a radio-telemetric system (RhemaLabortechnik, Hofheim, Germany, using 40 Megahertz as carrier frequency) and were amplified and processed as described earlier to give power spectra of $0.25 \mathrm{~Hz}$ resolution. In short, after automatic artefact rejection signals were collected in sweeps of $4 \mathrm{~s}$ duration and Fast Fourier transformed using a Hanning window. Sampling frequency was $512 \mathrm{~Hz}$. Four values were averaged to give a final sampling frequency of $128 \mathrm{~Hz}$, well above the Nyquist frequency [7]. The resulting electrical power spectra were divided into 7 pre-defined frequency ranges (delta: 1.5 - 4.0 Hz; theta: 4.25 - $6.75 \mathrm{~Hz}$; alpha 1: 7.00 - $9.50 \mathrm{~Hz}$; alpha 2: 9.75 - $12.25 \mathrm{~Hz}$; beta 1a: 12.50 - 15.00

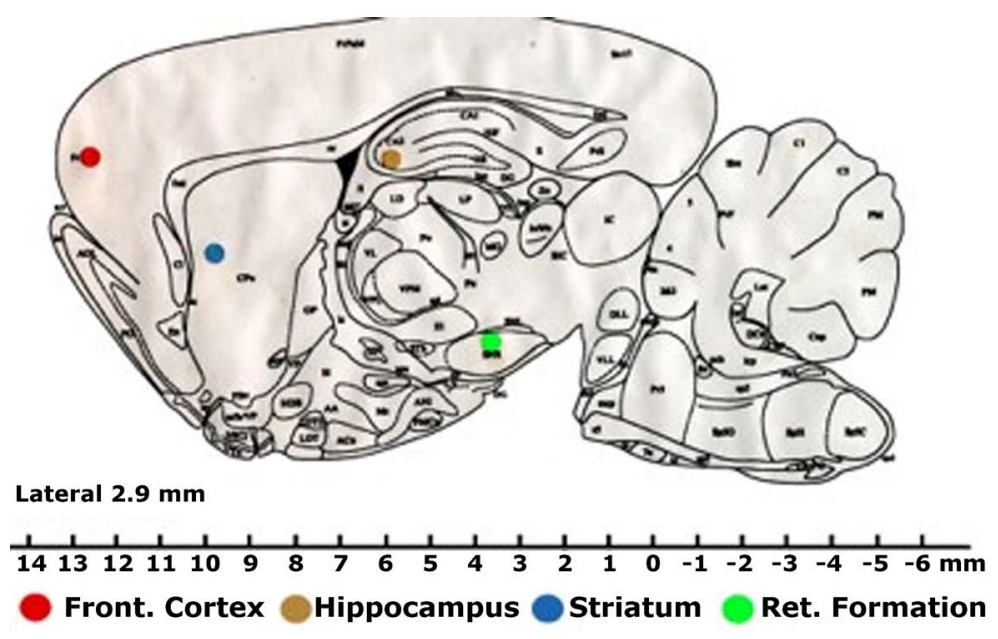

Figure 1. Locations for recording local field potentials (LFP). 


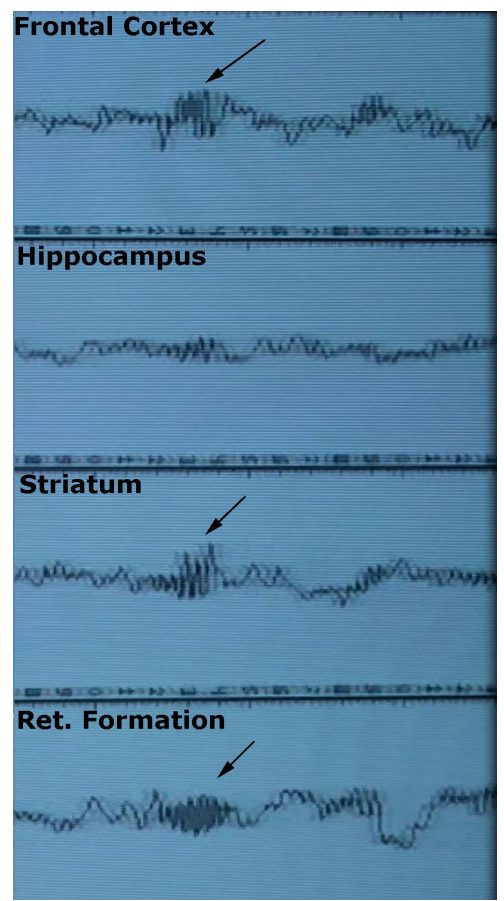

Figure 2. Documentation of online recording of a rat during active movement. Local field potential recorded from Cortex, Hippocampus, Striatum and Reticular formation. Arrows mark slow gamma.

Hz; beta 1b: 15.25 - $17.75 \mathrm{~Hz}$; beta 2: 18.00 - $34.25 \mathrm{~Hz}$; gamma: 34.50 - $81.00 \mathrm{~Hz}$ ). Spectra were averaged in steps of $3 \mathrm{~min}$ each and displayed on-line. In an off-line procedure spectra were averaged to give longer periods of 15 minutes for further analysis and data documentation. Statistical evaluation was done by means of Pearson correlation analysis.

Animals were housed in single cages with food and water ad lib. Maintenance food (Nohrlin $10 \mathrm{H}$ ) was achieved from Altromin Spezialfutter GmbH \& Co. KG in Lage, Germany. Animals were held during an inverted light-dark cycle (day $=$ darkness) in order to achieve stabile recording during their active phase [5]. The research was conducted in accordance with the internationally accepted principles for laboratory animal use and care as testified by authority allowance from Regierungspräsidium Giessen\# V54-19c2015h01 dated 17.03.2015 including vote of ethics committee.

\section{Results}

During on line documentation of local field potentials of 10 rats (three rats at the same time) we observed fast oscillations in a burst like manner, which appeared predominantly within the striatum (Figure 2) and to a clearly less extent in the frontal cortex and reticular formation. Nearly no such bursts of activity were detected in the hippocampus. However, they emerged always in a synchronized manner. These oscillations only occurred during movement of the animals and were never seen when the animal was immobile (Figure 3(a)). We therefore took a closer look at these potentials. As documented in Figure 3(b) these bursts contained high frequency activity for 1 or 2 seconds predominantly in the striatum during movement.

In order to further analyze this activity sweeps of $4 \mathrm{~s}$ duration a FFT was calculated for these representative signals as documented in Figure 3(a) and Figure 3(b). Comparing the power spectrum of the LFP of identical timings within the four brain regions during movement revealed a peak of gamma activity in the range of about $47 \mathrm{~Hz}$ as documented in Figure 4 for all brain areas on the right side. This gamma activity was only present periodically in all brain areas except for the hippocampus when the animals moved. During immobility no gamma activity between 40 and $50 \mathrm{~Hz}$ was detected (Figure 4, left side).

In order to obtain more proof for the appearance of gamma activity during movement, periods of 15 mins du- 


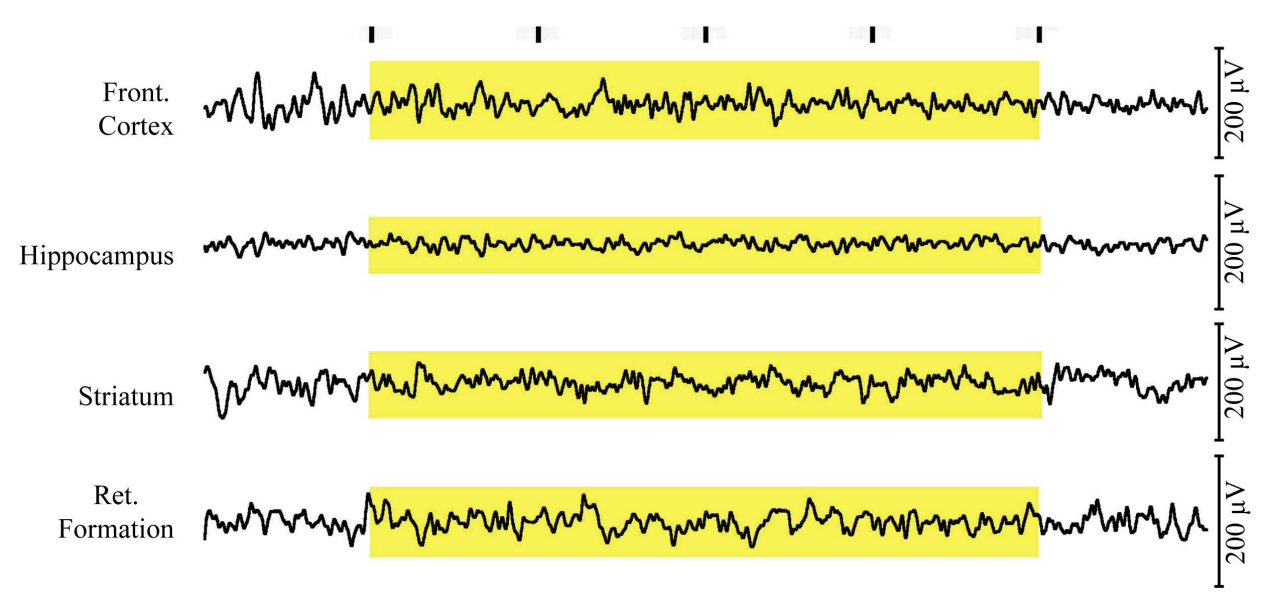

(a)

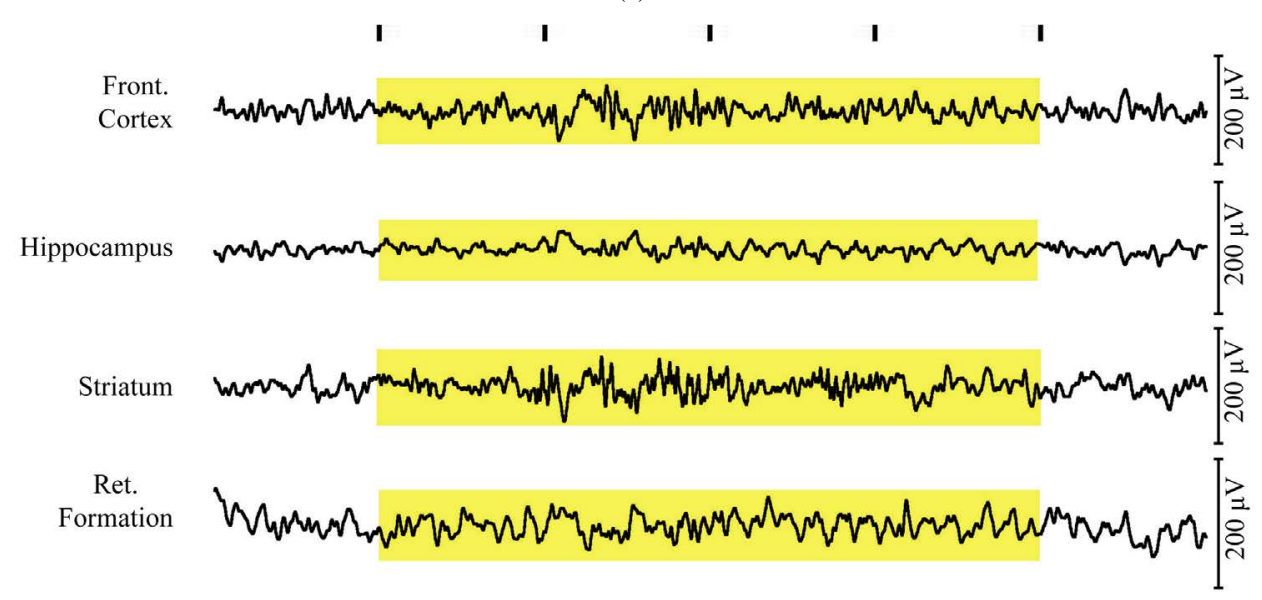

(b)

Figure 3. (a): Local field potentials of 4 s duration (marked yellow) in the immobile rat. (b): Local field potentials of $4 \mathrm{~s}$ duration (marked yellow) in the active rat. Please note slow gamma activity between 40 and $50 \mathrm{~Hz}$ mainly in the striatum and frontal cortex.

ration were fed into spectral analysis for each brain region during a highly active and a mostly immobile status. As expected, only the spectra from the active period contained larger amounts of gamma power peaking at about $47 \mathrm{~Hz}$. Spectra from an active and an immobile period of 15 minutes duration are documented in Figure 5.

Quantitation of movement at 15 minutes intervals was measured by a video-based motion detector for the whole duration of the experiment of 5.75 hours. The period with the highest motion was then compared to the one with the lowest motion with respect to gamma activity. The result from the highest motion was divided by the result obtained for the lowest motion to give a quotient. Data are given in Table 1 for each single rat. As one can see from the table, values in the striatum were considerably higher than in any other area $(\mathrm{P}<0.002)$. According to a correlation analysis, data from striatum were related to those obtained in the frontal cortex and reticular formation but not to those obtained from the hippocampus. However, data from the frontal cortex were not directly related to those from the reticular formation.

Finally, the whole time course of movement changes and spectral gamma activity was fed into a correlation analysis according to Pearson for each of the ten rats. For all rats a high correlation was observed with respect to data recorded from the striatum. The data including regression lines are given in Figure 6 and Figure 7 for all 10 animals.

\section{Discussion}

Local field Potentials are an important part of the brain’s communication structure. Quantitative assessment can 

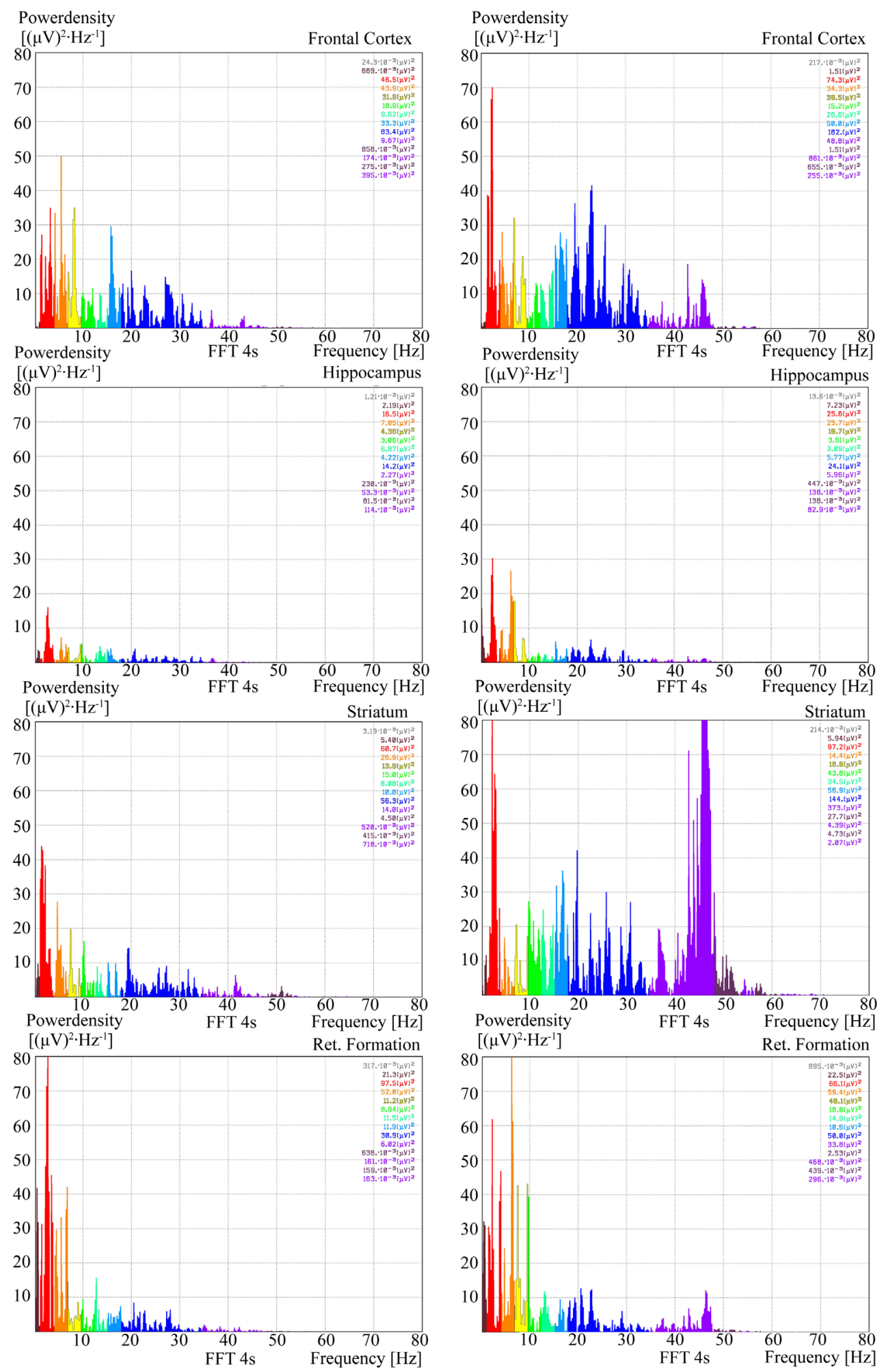

(a)

(b)

Figure 4. (a): Spectral analysis of data obtained from an immobile rat (4 s duration from Figure 3 ) with respect to all four brain regions (upper right). (b): Spectral analysis of LFP in the active rat (data from Figure 3). Note gamma activity (peaking between 40 and $50 \mathrm{~Hz}$ ) in all brain areas except for the hippocampus. 
W. Dimpfel, L. Schombert
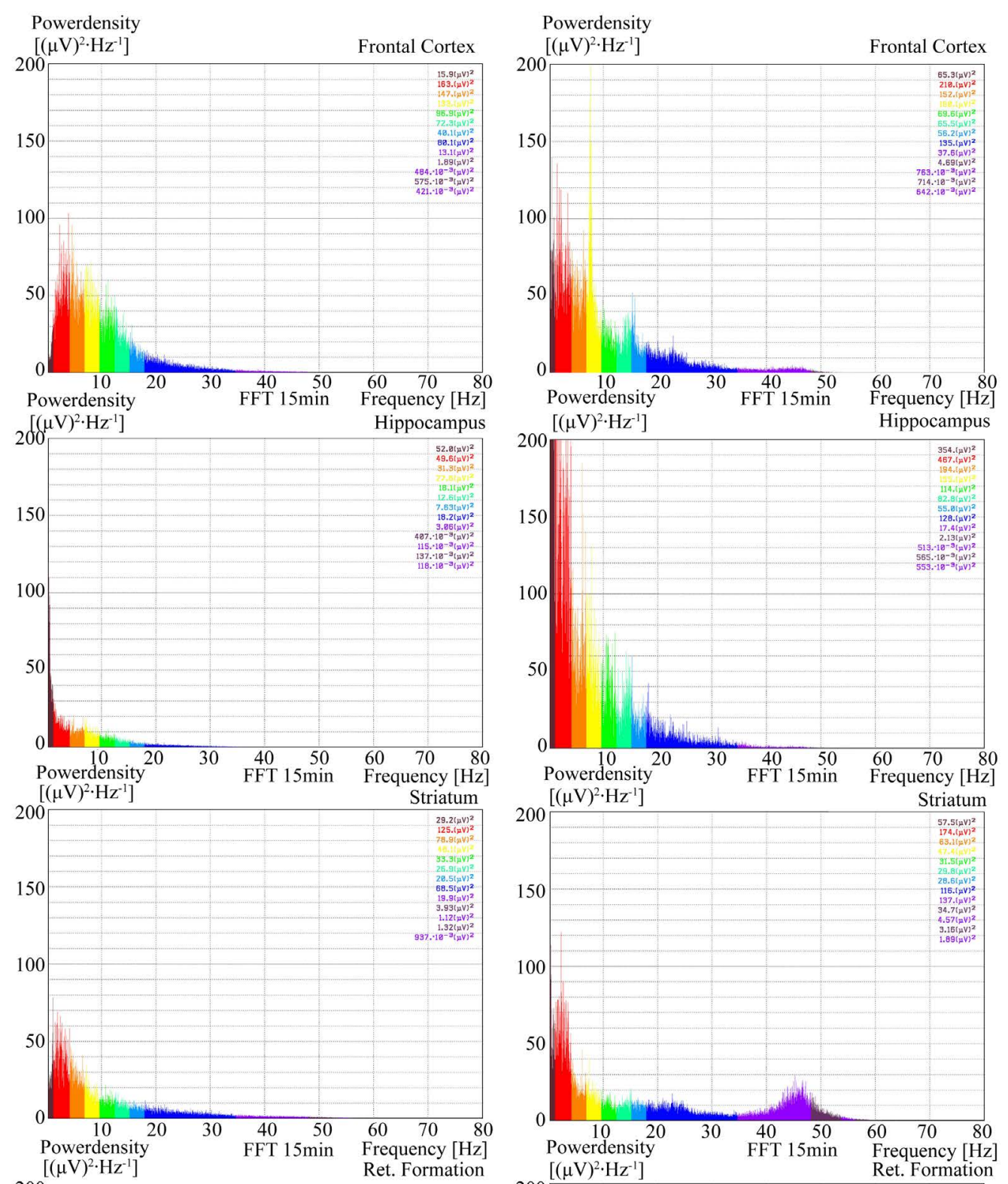

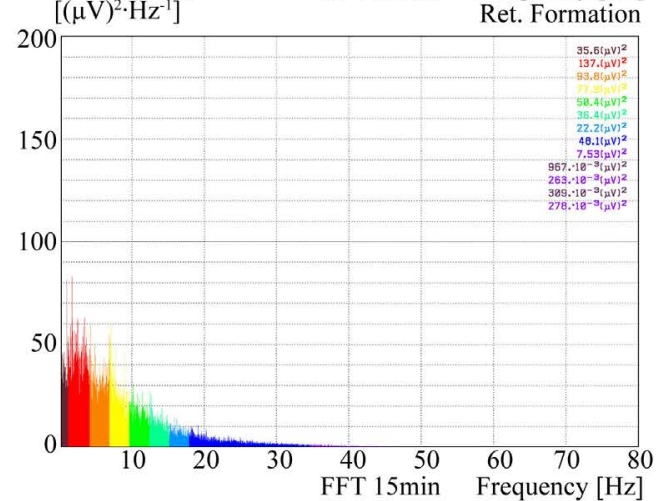

(a)

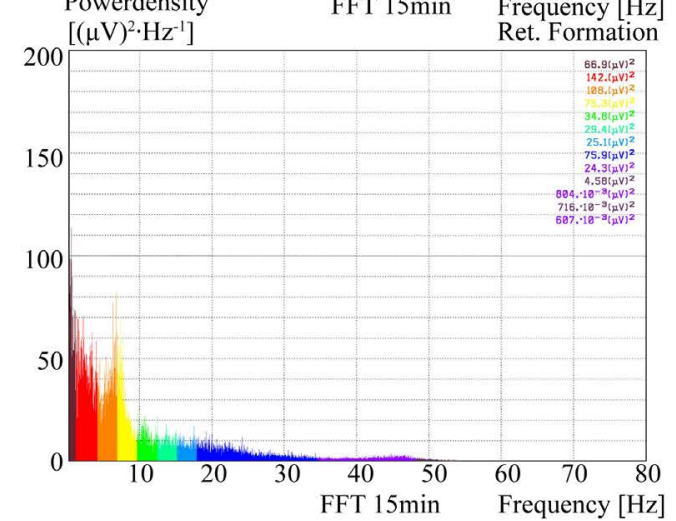

(b)

Figure 5. Spectral power in the immobile (a) and active rat (b) averaged for 15 minutes. Data are presented for all four brain areas. Please note strongest gamma power $(40-50 \mathrm{~Hz})$ in the striatum of the active rat. 
Table 1. Relationship between gamma activity in the most active $15 \mathrm{~min}$. period and the $15 \mathrm{~min}$. period with the lowest movement in each rat. The quotient is given for each brain region. Values of the striatum differed highly significant from the other brain regions with $\mathrm{P}<0.002$ (Sign Test). Rank correlations are given for comparison between brain regions significantly. (ST = striatum, FC = frontal cortex, HIP = hippocampus, $\mathrm{RF}=$ reticular formation) as documented in the lower part of the table.

\begin{tabular}{|c|c|c|c|c|}
\hline \multicolumn{5}{|c|}{ Quotient High Gamma Activity /Low Gamma Activity } \\
\hline Rat \# & Frontal Cortex & Hippocampus & Striatum & Ret. Formation \\
\hline 1 & 2.00 & 1.09 & 4.07 & 2.23 \\
\hline 2 & 1.56 & 0.99 & 1.93 & 1.21 \\
\hline 3 & 1.66 & 1.20 & 3.40 & 1.44 \\
\hline 4 & 2.18 & 1.31 & 7.04 & 1.99 \\
\hline 5 & 1.60 & 1.43 & 4.50 & 1.67 \\
\hline 6 & 1.26 & 1.27 & 4.78 & 2.28 \\
\hline 7 & 2.00 & 1.11 & 6.43 & 1.63 \\
\hline 8 & 2.03 & 1.15 & 4.91 & 1.80 \\
\hline 9 & 1.34 & 1.23 & 1.96 & 1.11 \\
\hline 10 & 1.13 & 1.65 & 3.07 & 1.87 \\
\hline mean & 1.68 & 1.24 & 4.21 & 1.72 \\
\hline \multirow[t]{5}{*}{ SEM } & 0.12 & 0.06 & 0.54 & 0.12 \\
\hline & $\mathrm{FC}<>\mathrm{HIP}$ & $\mathrm{HIP}<>\mathrm{RF}$ & $\mathrm{ST}<>\mathrm{FC}$ & $\mathrm{ST}<>\mathrm{RF}$ \\
\hline & $\mathbf{r}=-\mathbf{0 . 3}$ & $r=0.3$ & $\mathbf{r}=0.7$ & $r=0.5$ \\
\hline & \multicolumn{2}{|c|}{$\mathrm{FC}<>\mathrm{RF}$} & \multicolumn{2}{|c|}{$\mathrm{ST}<>\mathrm{HIP}$} \\
\hline & \multicolumn{2}{|c|}{$r=0.01$} & \multicolumn{2}{|c|}{$\mathrm{r}=0.2$} \\
\hline
\end{tabular}

be obtained by fast transformation of the raw data according to Fourier (FFT). For interpretation the whole power spectrum resulting from this transformation is split into several windows or ranges. Historically, these frequency ranges are labeled according to Greek letters, namely delta, theta, alpha, beta and gamma from the lowest frequency to the highest, respectively. Unfortunately, there is no common understanding on the borderlines of these ranges and ranges are also subdivided into smaller subranges (s. methods). However, for some of the frequency ranges as we defined them in earlier publications a relationship to neurotransmitter activities was discovered. The present experiments deal with the fast gamma frequencies generally defined as frequencies above $35 \mathrm{~Hz}$. These fast frequencies have been related to a large number of behavioral situations like movement, memory, attention, decision making in animals and humans. Gamma activity has been recorded from many brain areas in animals and humans. For example, coherent gamma oscillations seem to coordinate amygdalostriatal interactions during learning in cats and might facilitate synaptic plasticity [8]. There is also evidence in humans that not only motion itself but also perception of biological motion relates to gamma activity, in this case so-called high gamma frequencies $(70-100 \mathrm{~Hz})$ [9]. High gamma oscillations $(70-94 \mathrm{~Hz})$ have also been recorded from nucleus accumbens in rats during induction of stereotype behavior by methamphetamine [10]. In this case a relationship was detected between this high gamma activity and the cannabinoid receptor CB1.

Low gamma oscillations ( $30-56 \mathrm{~Hz}$ ) have been related in healthy subjects and schizophrenic patients to dorso-lateral prefrontal levels of GABA at rest and during a memory task [11].

Our recording relates to frontal cortex, hippocampus, striatum and reticular formation in the freely behaving rat as documented in numerous publications. The result from this experimental series provides strong evidence that so-called low gamma activity in the range between 40 and $50 \mathrm{~Hz}$ (peaking at about $47 \mathrm{~Hz}$ ) is related to action and movement. Already a small movement of the head was accompanied by bursts of gamma activity, pre- 

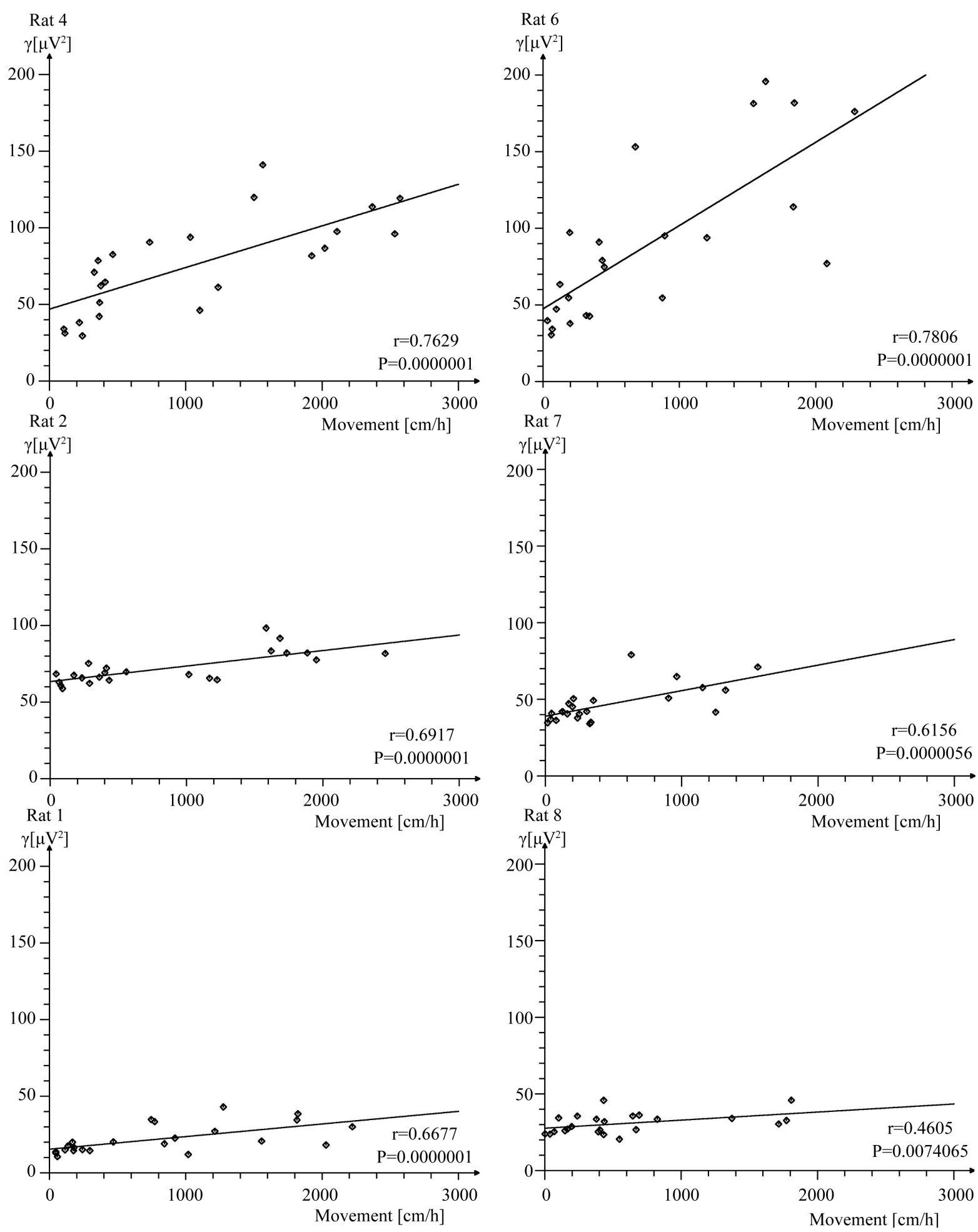

Figure 6. Correlation analysis and regression between gamma activity and movement from 6 different single rats based on data recorded from the striatum for 5.75 hours. Correlation analysis according to Pearson with coefficient $\mathrm{r}$ and statistical power. Correlation coefficients and p values are given at the right lower corner of the images.

dominantly in the striatum, but also visible in frontal cortex and reticular formation, least or not at all in the hippocampus. Since motion was independently measured by an external video-based method, a correlation be- 

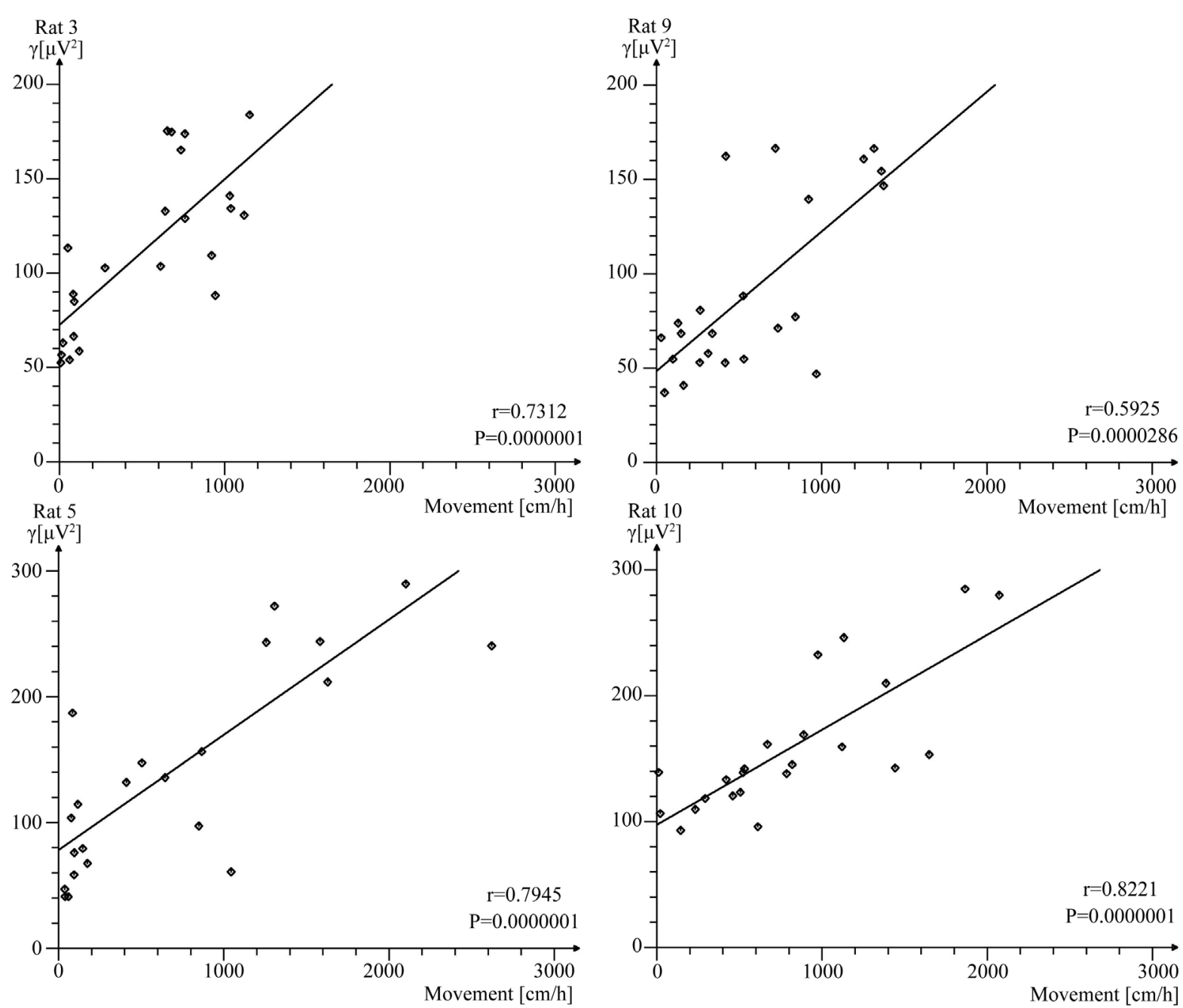

Figure 7. Correlation analysis and regression between gamma activity and movement from 4 different single rats based on data recorded from the striatum for 5.75 hours. Correlation analysis according to Pearson with coefficient $\mathrm{r}$ and statistical power. Correlation coefficients and p values are given at the right lower corner of the images.

tween motion and gamma activity became possible. The result of the correlation analysis revealed that all 10 rats followed for 5.75 hours showed a very high correlation. We therefore conclude from these experiments that this slow gamma activity of the field potentials is not only related to movement, but possibly part of the neuronal coding of movement as suggested already by [1]. To our knowledge, first analysis of such neuronal oscillations was reported in 1994 [12]. Local field potential oscillations (defined as gamma band (20 - $80 \mathrm{~Hz})$ ) were related to movements as early as 1998 drawing a similar conclusion: “....must reflect a global process active in conjunction with motor planning or preparatory functions" [13].

\section{References}

[1] Igarashi, J., Isomura, Y., Arai K., Harukuni, R. and Fukai T. (2013) A $\theta-\gamma$ Oscillation Code for Neuronal Coordination during Motor Behavior. The Journal of Neuroscience, 33, 18515-18530. http://dx.doi.org/10.1523/JNEUROSCI.2126-13.2013

[2] Masimore, B., Kakalios, J. and Redish, A.D. (2004) Measuring Fundamental Frequencies in Local Field Potentials. Journal of Neuroscience Methods, 138, 97-105. http://dx.doi.org/10.1016/j.jneumeth.2004.03.014

[3] Masimore, B., Schmitzer-Torbert, N.C., Kakalios, J. and Redish, A.D. (2005) Transient Striatal Gamma Local Field Potentials Signal Movement Initiation in Rats. Neuroreport, 16, 2021-2024.

http://dx.doi.org/10.1097/00001756-200512190-00010 
[4] Van der Meer, M.A. and Redish, A.D. (2009) Low and High Gamma Oscillations in Rat Ventral Striatum Have Distinct Relationships to Behavior, Reward, and Spiking Activity on a Learned Spatial Decision Task. Frontiers in Integrative Neuroscience, 3, 9.

[5] Dimpfel, W. (2003) Preclinical Data Base of Pharmaco-Specific Rat EEG Fingerprints (Tele-Stereo-EEG). European Journal of Medical Research, 8, 199-207.

[6] Paxinos, G. and Watson, C. (1982) The Rat Brain in Stereotactic Coordinates. Academic Press, New York.

[7] Dimpfel, W., Spüler, M., Koch, R. and Schatton, W. (1987) Radioelectroencephalographic Comparison of Memantine with Receptor-Specific Drugs Acting on Dopaminergic Transmission in Freely Moving Rats. Neuropsychobiology, 18, 212-218. http://dx.doi.org/10.1159/000118420

[8] Popescu, A.T., Popa, D. and Paré, D. (2009) Coherent Gamma Oscillations Couple the Amygdala and Striatum during Learning. Nature Neuroscience, 12, 801-807.

[9] Darvas, F., Murias, M. and Rao, R.P.N. (2013) Localized High Gamma Motor Oscillations Respond to Perceived Biological Motion. Journal of Clinical Neurophysiology, 30, 299-307. http://dx.doi.org/10.1097/WNP.0b013e3182872f40

[10] Morra, T., Glick, S.D. and Cheer, J.F. (2012) Cannabinoid Receptors Mediate Metamphetamine Induction of High Gamma Oscillations in the Nucleus Accumbens. Neuropharmacology, 63, 565-574. http://dx.doi.org/10.1016/j.neuropharm.2012.04.036

[11] Chen, C-M.A., Stanford, A.D., Mao, X., Abi-Dargham, A., Shungu, D.C., Lisanby, S.H., Schroeder, C.E. and Kegeles, L.S. (2014) GABA Level, Gamma Oscillation, and Working Memory Performance in Schizophrenia. NeuroImage: Clinical, 4, 531-539. http://dx.doi.org/10.1016/j.nicl.2014.03.007

[12] Gray, C.M. (1994) Synchronous Oscillations in Neuronal Systems: Mechanism and Functions. Journal of Computational Neuroscience, 1, 11-38. http://dx.doi.org/10.1007/BF00962716

[13] Donoghue, J.P., Sanes, J.N., Hatsopoulos, N.G. and Gaál, G. (1998) Neural Discharge and Local Field Potemtial Oscillations in Primate Motor Cortex During Voluntary Movements. Journal of Neurophysiology, 79, 159-173. 